\section{Uncompromised intraoral scanning}

Step into a new generation of intraoral excellence when you choose the CS 3700 intraoral scanner from Carestream

Dental.

Building on the success of its predecessor, the CS 3700 now offers even faster scanning times alongside a slew of state-of-the-art features that have been designed to make intraoral scanning better and easier than ever before.

For example, when taking an image with the CS 3700 , shade match data is instantly recorded in the digital file. This helps laboratories to create restorations with optimal aesthetics.

The scanner also champions adaptability - CS ScanFlow software lets you pursue any indication from just a single scan!

With the CS 3700 , the power is in your hands.

For more information, contact Carestream Dental on 08001699692 or visit www.carestreamdental.co.uk.

\section{No better time to join this professional academy}

The BACD is a vibrant community of dentists, students, and dental technicians.

A world-leading authority on ethical cosmetic dentistry, the BACD has always been forward thinking when it comes to making every patient feel comfortable and informed. It has comprehensive protocols for treatment planning, indispensable for the challenges ahead.

Patients can feel safe with BACD dentists. Members also have access to unparalleled learning opportunities, including a recently launched online learning portal, to ensure they are up-todate on the latest research and best practice.

To be part of the BACD, and to find out about all its benefits, visit the website today.

For further enquiries about the British Academy of Cosmetic Dentistry visit www.bacd.com.

\section{Preparing for new restorative challenges}

The risk of transmission of infection during aerosol generating procedures (AGPs) has long been a concern to the dental profession, and something that's been highlighted during the COVID-19 pandemic.

AGPs play a significant part in daily working practice, notably the use of highspeed turbines for restorative procedures, extractions and incisions. However, current guidance ${ }^{1}$ to the profession advises that all urgent dental procedures, including oral examinations, should be treated as aerosol-generating.

GC's simple GIC restorative systems means you can provide patients with immediate, definitive clinical solutions - all with a minimal amount of aerosol generation.

Broken cusps, cavities or fractured teeth can be repaired swiftly and safely using EQUIA Forte bulk fill glass hybrid restorative system the ideal solution for posterior restorations.

- Suitable for all age groups

- No etching or bonding

- High compressive strength

- Moisture tolerant

- Suitable for Class I, Class $\mathrm{II}^{2}$ and Class V restorations.

Patients seeking immediate relief from sensitive teeth or unchecked caries need protection fast. Fuji TRIAGE effectively works as a visible, semi-permanent 'tooth plaster' to provide temporary relief until they can return for a permanent restoration.

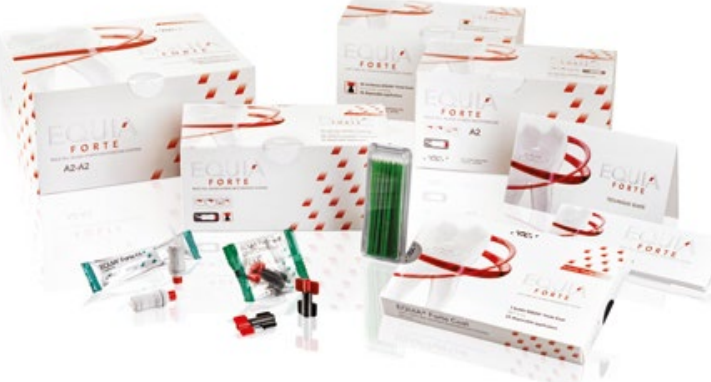

- White or translucent pink GIC with chemical bond to tooth

- No etching or bonding

- Low viscosity to penetrate pits and fissures

- Provides instant, long-lasting relief from hypersensitivity

- Available in capsules or as a powder and liquid.

For further information, get in touch with GC UK today to request a virtual meeting with one of the team and discuss GC's reduced AGP solutions. Contact GC UK Ltd on 01908 218999, email info.uk@gc.dental or visit www.gceurope.com.

\section{References}

1. BAOMS. Guidance PPE for patients with emergency oral and dental problems of unknown COVID Status. 25 March 2020. Available at: https://www.baoms.org. uk/_userfiles/pages/files/professionals/covid_19/ baoms_baos_covid_advice_update_25_march_2020 final.pdf (accessed June 2020).

2. Friedl K, Hiller K-A, Friedl K-H. Clinical performance of a new glass ionomer based restoration system: a retrospective cohort study. Dent Mater 2011; 27: 1031-1037.

\title{
Ultimate control with self-litigating brackets
}

Designed with both orthodontists and patients in mind, Clarity Ultra self-litigating brackets from $3 \mathrm{M}$ stand out from the crowd due to a number of innovative features.

Featuring a wide ceramic door that assists rotational control, the brackets are also durable and made from fine-grained alumina to promote strength. This helps ensure they are an effective, long-lasting solution.

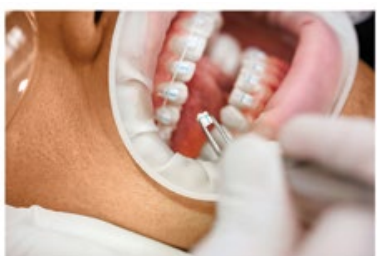

The brackets are also versatile to both complex and simple orthodontic needs, meaning that they are a wonderful option for patients of all ages too.

Find out more about how the design of the Clarity Ultra self-litigating brackets can benefit you and your patients by contacting 3M Oral Care.

For more information, call 0845873 4066 or visit http://solutions.3m. co.uk/wps/portal/3M/en_GB/ orthodontics_EU/Unitek/

$3 \mathrm{M}$ and Clarity are trademarks of the $3 \mathrm{M}$ Company. 